\title{
La gérance des antibiotiques : un rôle important du domaine de la pharmacie?
}

\author{
par Christine M Bond
}

$\mathrm{C}$ haque année, des milliers de personnes meurent des suites d'infections causées par des bactéries résistantes aux antibiotiques. L'incidence croissante de la résistance aux antibiotiques a été décrite comme une menace de santé publique mondiale. Des efforts concertés sont donc nécessaires pour s’attaquer au problème. Dans le présent numéro du JCPH, Wu et collab. ${ }^{1}$ présentent une petite vérification de l'utilisation des antibiotiques dans un établissement de soins de longue durée. La vérification a permis de relever une dépendance excessive à l'égard des céphalosporines de troisième génération et des fluoroquinolones ainsi que le recours aux diagnostics de présomption en l'absence d'antibiogramme. Bien que l'article ne décrive qu'une vérification mineure dans un seul établissement, il montre que, malgré que l'on s'entende sur l'existence du problème de la résistance aux antibiotiques, un fossé demeure entre la connaissance et la pratique actuelle.

Les antibiotiques jouent un rôle central dans l'amélioration de la santé de la population depuis qu'ils ont pour la première fois servi de traitement dans les années 1940. En effet, ils ont eu un impact à ce point considérable sur le traitement des maladies infectieuses que l'on a affirmé qu'ils permettraient de « faire des maladies infectieuses une chose du passé $\aleph^{2}$. Nous savons aujourd'hui que tel n'est pas le cas. L'usage croissant des antibiothérapies en médecine a mené à une dépendance excessive chez les patients et à une surprescription par les professionnels. Ces problèmes, combinés à celui de l'utilisation d'antibiotiques dans l'élevage, se sont traduits par l'apparition de la résistance aux antibiotiques, ce qui a par conséquent réduit l'efficacité de l'arsenal actuel des traitements anti-infectieux. Parallèlement, le taux de développement de nouveaux antibiotiques a diminué, ne permettant pas de supplanter les traitements caducs sur l'étagère de l'officine. En effet, seulement deux nouvelles classes d'antibiotiques (les oxazolidinones et les lipopeptides cycliques) ont été commercialisées au cours des 30 dernières années ${ }^{3}$. Les raisons qui expliquent la rareté des nouveaux antibiotiques résident dans les nombreux défis rattachés au développement de médicaments de nos jours. Parmi ceux-ci, on compte l'augmentation du temps nécessaire à la commercialisation d'un nouveau médicament, les défaites coûteuses en cours de projet ainsi que l'ensemble des investisse- ments financiers nécessaires. Par ailleurs, contrairement à la situation dont jouissent les médicaments employés pour traiter les maladies chroniques, le retour sur investissement des antibiotiques n'est pas intéressant et tout nouvel antibiotique ne possède qu'une faible viabilité commerciale. En général, les antibiotiques servent de traitements à court terme et mènent normalement à la guérison. De plus, tout nouvel antibiotique commercialisé sera très certainement accompagné de directives en limitant l'emploi aux seuls cas où tous les autres traitements ont échoué. Donc, bien que des approches novatrices pour découvrir de nouveaux antibiotiques soient étudiées (notamment trouver de nouvelles sources de molécules dans le règne végétal ou animal et rechercher de nouveaux mécanismes d'action [dont l'utilisation de bactériophages ou d'anticorps génospécifiques] $]^{4}$ ), elles représentent, au mieux, seulement des solutions à long terme.

Comme les chances de découvrir de nouveaux antibiotiques dans un avenir proche sont faibles, il appartient à la population et aux professionnels de la santé de ménager ceux qui existent à l'aide de ce que l'on appelle la gérance des antibiotiques (ou gérance des antimicrobiens). Ce terme englobe une gamme de gestes, notamment : encourager les programmes de promotion de l'hygiène et du lavage des mains ainsi que promouvoir des pratiques de prescription adéquates (c'est-à-dire prescrire un antibiotique seulement lorsqu'il est nécessaire et, le cas échéant, s'assurer qu'il s'agit du bon médicament et que la dose ainsi que la durée du traitement soient exactes). Au niveau organisationnel, il se traduit par des systèmes de surveillance et de directives visant à favoriser une approche fondée sur des données probantes 5 . En ce qui touche les patients, il s'agit « du choix, de la posologie et de la durée de traitement qui produisent les meilleurs résultats cliniques pour le traitement ou la prévention de l'infection de même qu'une toxicité minimale pour le patient et une répercussion minimale sur une résistance ultérieure $»^{6}$.

Ce devrait maintenant être un fait bien connu - par les professionnels si ce n'est par le grand public - que bon nombre d'infections, et pas seulement les infections virales, ne nécessitent pas d'antibiothérapie. Par exemple, des données probantes appuient l'emploi d'un traitement symptomatique plutôt que 
celui d'un antibiotique pour traiter les maladies spontanément résolutives comme l'otite moyenne, car dans de tels cas une antibiothérapie n'a que peu d'effet sur la durée des symptômes associés ${ }^{7}$. L'utilisation abusive d'antibiotiques dans les milieux des soins primaires est en effet ce qui a conduit à l'apparition de la résistance aux antibiotiques dans la communauté et celle-ci demeure inapparente jusqu'à ce que le patient soit admis dans un hôpital ou un établissement de soins de longue durée.

Il est généralement admis que les prescripteurs n'adhèrent pas toujours aux pratiques fondées sur des données probantes. Ils cherchent à traiter l'individu plutôt qu'à considérer les effets à long terme du traitement sur ce patient (lorsqu'une résistance aux antibiotiques est présente chez un individu, elle peut durer plus d'un $\mathrm{an}^{8}$ ) ou sur la population. Les pharmaciens exercent habituellement une fonction de second plan quant à la prescription d'antibiotiques aux patients; ils sont donc en mesure d'offrir aux prescripteurs des conseils professionnels objectifs. En tant qu'experts dans le domaine des médicaments, les pharmaciens sont bien placés pour diriger le programme de gérance des antibiotiques, pas seulement en réalisant des vérifications et en attirant l'attention sur un problème, mais en influençant les décisions importantes liées à la prescription. En fait, on a déjà vanté le rôle du pharmacien au sein des équipes de gérance des antibiotiques ${ }^{9-11}$. Mais il n'y a malheureusement eu que peu de recherche dans ce domaine et ce rôle pour les pharmaciens n'est pas soutenu par des données empiriques de qualité.

Lanalyse systématique réalisée par Kaki et collab. ${ }^{12}$ (citée par Wu et collab. ${ }^{1}$ ) n'est pas facilement généralisable, car elle n'aborde que des articles concernant les soins intensifs. Elle a par contre montré que la gérance des antibiotiques pouvait réduire l'utilisation des antibiotiques, les coûts qui y sont associés ainsi que la durée des traitements et pouvait mener à un emploi plus adéquat de ces médicaments, à une diminution des cas d'événements indésirables et à moins d'utilisation à long terme. Il est d'ailleurs important de noter que, selon les résultats de l'analyse, la gérance des antibiotiques ne se traduit pas par une augmentation des taux d'infections nosocomiales, de la durée du séjour ou des taux de mortalité. Par contre, les auteurs ne mentionnent nullement le rôle des pharmaciens dans l'ensemble des interventions décrites.

Une étude récente, réalisée au Royaume-Uni ${ }^{13}$ et échelonnée sur une période de 18 mois, a produit des résultats encourageants sur les effets positifs d'un programme-conseil de vérification et de recommandations dirigé par des pharmaciens qui visait à améliorer les habitudes de prescription des antibiotiques. Le programme s'appliquait surtout à resserrer l'observance des lignes directrices et à consigner l'indication thérapeutique dans le dossier médical. Malgré les limites de cette étude qui a été réalisée dans un seul milieu et selon un modèle avant-après, ce rapport confirme mon hypothèse : les pharmaciens auront un rôle important à jouer dans la lutte contre cette menace mondiale qui nous touche tous. D'autres rapports isolés semblables étayent cette hypothèse ${ }^{2}$, mais davantage de preuves issues d'essais cliniques à répartition aléatoire sont nécessaires pour démontrer que la gérance des antibiotiques devrait normalement revenir aux pharmaciens. Enfin, que vous travailliez dans le domaine des soins primaires, intermédiaires, secondaires, tertiaires ou à long terme, nous vous prions d'aider à recueillir ces preuves!

[Traduction par l'éditeur]

Références

1. Wu LDY, Walker S, Elligsen M, Palmay L, Simor A, Daneman N. Antibiotic use and need for antimicrobial stewardship in long-term care. Can J Hosp Pharm. 2015;68(6):445-9.

2. Upshur R. Ethics and infectious disease [compte rendu]. Bull World Health Organ. 2008;86(8):654. Aussi publié au : www.who.int/bulletin/volumes/86/ 8/08-056242/en/.

3. Coates A, Halls G, Hu Y. Novel classes of antibiotics or more of the same? Br J Pharmacol. 2011;163(1):184-94.

4. New medicines, better medicines, better use of medicines: a guide to the science underpinning pharmaceutical practice. Londres (Royaume-Uni) : Royal Pharmaceutical Society; 2014. Publié au : www.rpharms.com/promotingpharmacy-pdfs/nmbmbu---full-report.pdf. Consulté le 28 septembre 2015.

5. Tamma PD, Cosgrove SE. Antimicrobial stewardship. Infect Dis Clin North Am. 2011;25(1):245-60.

6. Gerding DN. The search for good antimicrobial stewardship. Jt Comm J Qual Improve. 2001;27(8):403-4.

7. Little P, Gould C, Moore M, Warner G, Dunleavy J, Williamson I. Predictors of poor outcome and benefits from antibiotics in children with acute otitis media: pragmatic randomised trial. BMJ. 2002;325(7354):22.

8. Costelloe C, Metcalfe C, Lovering A, Mant D, Hay A. Effect of antibiotic prescribing in primary care on antimicrobial resistance in individual patients: systematic review and meta-analysis. BMJ. 2010;340:c2096.

9. Dellit TH, Owens RC, McGowan JE Jr, Gerding DN, Weinstein RA, Burke JP, et collab. Infectious Diseases Society of America and the Society for Healthcare Epidemiology of America guidelines for developing an institutional program to enhance antimicrobial stewardship. Clin Infect Dis. 2007;44(2):159-77.

10. Gestion de l'utilisation des antimicrobiens : énoncé sur le rôle du pharmacien. Ottawa (ON) : Société canadienne des pharmaciens d'hôpitaux; 2014. Publié au : www.cshp.ca/dms/dmsView/1_1_Antimicrobial-StewardshipStatement-FR-Final.pdf. Consulté le 30 septembre 2015.

11. ASHP statement on the pharmacist's role in antimicrobial stewardship and infection prevention and control. Bethesda (MD) : American Society of Health-System Pharmacists; Publié au : www.ashp.org/doclibrary/ bestpractices/specificstantimicrob.aspx. Consulté le 30 septembre 2015.

12. Kaki R, Elligsen M, Walker S, Simor A, Palmay L, Daneman N. Impact of antimicrobial stewardship in critical care: a systematic review. J Antimicrob Chemother. 2011;66(6):1223-30.

13. Roberts E, Dawoud DM, Hughes DA, Cefai C. Evaluation of a consultant audit and feedback programme to improve the quality of antimicrobial prescribing in acute medical admissions. Int J Pharm Pract. 2015;23(5):333-9.

Christine M Bond, B. Pharm., Ph. D., M. Ed., travaille au Pharmacy, Centre of Academic Primary Care de I'Université d'Aberdeen, Foresterhill, Aberdeen, Écosse. Elle est également rédactrice adjointe du JCHP.

Intérêts concurrents : Christine Bond est rédactrice en chef de I'International Journal of Pharmacy Practice et est membre des comités de rédaction du Journal of Self Care, du Prescriber, du Pilot and Feasibility Studies et du Journal of Pharmaceutical Health Services Research. Elle préside aussi l'Expert Advisory Panel for Science de la Royal Pharmaceutical Society.

\section{Address correspondence to:}

De Christine M Bond

Pharmacy

Centre of Academic Primary Care

Room 1.123

Polwarth Building West Block

Foresterhill, Aberdeen AB25 2ZD

Scotland

Courriel : c.m.bond@abdn.ac.uk 